\title{
Rosai - Dorfman Disease Masquerading As Sarcoma: A Case Report
}

\author{
Sudha P. Meena ${ }^{1}$, Priyanka Patangia ${ }^{2 *}$ and Naresh N. Rai ${ }^{1}$ \\ ${ }^{1}$ Department of Pathology, Government Medical College, Kota(India) \\ ${ }^{2}$ Department of Pathology, AIIMS, Bhopal(India)
}

\begin{abstract}
Rosai Dorfman disease known as sinus histiocytosis with massive lymphadenopathy is an uncommon benign systemic histo-proliferative disease which affects thecervical lymph nodes commonly. The characteristic histological feature is lymphophagocytosis(emperipolesis) which is the hallmark feature. Here, we report a case of 35 year old Indian female presenting as a mass on medial aspect of thigh appearing as soft tissue mass, which on cytological examination was misdiagnosed as malignant mesenchymal neoplasm but histopathological examination and immunohistochemistry confirmed the diagnosis. According to the literature reviewed no case of Rosai Dorfman disease with only ingunal lymphadenopathy was reported till date which was seen in present case. RDD is a rare inflammatory non neoplastic process that should be considered in the differential diagnosis of lympadenopathy and soft tissue tumor such as soft tissue sarcoma. The diagnosis of RDD is made on the basis of clinical suspicion and is confirmed by histopathology and immunohistochemistry
\end{abstract}

Keywords: Rosai-Dorfman Disease; Sinus Histiocytosis; Sarcoma; Immunohistochemistry

\section{Introduction}

Rosai-Dorman disease (RDD), also known as sinus histiocytosis with massive lymphadenopathy (SHML), was originally described by Destombes in 1965. Subsequently, it was characterized as a distinct clinicopathological disorder in 1969 by Rosai and Dorfman as a non-neoplastic, usually self-limiting disease of unknown cause. ${ }^{[1,2]}$

SHML most commonly presents as painless cervical lymphadenopathy, with frequent involvement of other lymph node groups like inguinal, axillary and mediastinal lymph node basins. ${ }^{[3]}$ In $30 \%$ cases extranodal sites are involved, such as skin, bone and breast. ${ }^{[2-4]}$ Associated clinical and laboratory features are fever, leukocytosis, elevated erythrocyte sedimentation rate and hypergammaglobulinemia. ${ }^{[4]}$ Although fatalities in patients who have SHML rarely have been reported, it is seen particularly in patients who have immunologic abnormalities. ${ }^{[5]}$ SHML usually follows a benign clinical course, often with spontaneous resolution of disease.

Histology and immunohistochemistry help differentiate RDD from malignant disorders such as lymphoma and Langerhans cell histiocytosis. Although lymphadenopathy can be significant and disfiguring, RDD is usually selflimiting and eventually recedes, making systemic therapy rarely required. ${ }^{[6]}$

Here, we report a case of 35yearold Indian female presenting with a mass on medial aspect of thigh appearing as soft tissue mass, which on cytological examination was misdiagnosed as malignant mesenchymal neoplasm but on histopathological examination and immunohistochemistry confirmed the diagnosis of Rosai Dorfman disease.

\section{Case History}

A 35 years old female reported to our hospital with a 2 month history of an enlarging left medial thigh mass. $\mathrm{Hb}-$ $9.1 \mathrm{~g} / \mathrm{dl}$, WBC count $-6.35 \times 10^{3} / \mathrm{uL}, \mathrm{N}-88.2 \%$, L-3.3\%, M-5.0\%, E- 3.3\% . PBF shows microcytic hypochromic red blood cells. Her medical history was not significant for hypertension, diabetes, hypercholesterolemia. On clinical examination a well defined, firm, nodular, deep subcutaneous swelling, with overlying skin appearing normal measuring $5 \times 4 \mathrm{~cm}$ in size. No cervical or axillary lymphadenopathy and hepatosplenomegaly seen.

On FNA of left thigh swelling (Figure 1) blood mixed aspirate was obtained. Smears were air dried and stained by Geimsa stain. On microscopic examination, it was reported as malignant mesenchymal neoplasm with possibility of malignant fibrous histiocytoma (inflammatory variant) (figure 2) . Patient underwent surgical excision for the same. On gross examination there was a well circumscribed and encapsulated firm grey white to tan coloured fleshy mass showing multiple nodules within subcutaneous tissue measuring $5 \times 4 \times 3 \mathrm{~cm}$ ((Figure 3$)$. There were no changes on the overlying skin.

Microscopic examination shows thickened capsule with dialated underlying subcapsular sinus along with marked 
dilatation of lymph sinuses [Figure 4 ], packed with histiocytic cells with vacuolated or foamy cytoplasm, lymphophagocytosis (emperipolesis) (Figure 5,6). Depending on this, the patient was suspected to be suffering from RDD (SHML). For confirmation of the diagnosis immunohistochemistry for S-100 was advised, which was strongly positive (Figure 7). Thus confirming the diagnosis of RDD.
On review of cytology smears, it revealed presence of diffusely distributed histiocytes throughout the smears (Figure 8). These cells had abundant pale cytoplasm. The nuclei showed fine chromatin and inconspicuous nucleoli. The cytoplasm of these histiocytes exhibited intact lymphocytes (emperepolesis). The background had mature lymphocytes, plasma cells, neutrophils and tingible body macrophages. This cytological features favours the diagnosis of Rosai Dorfman disease.

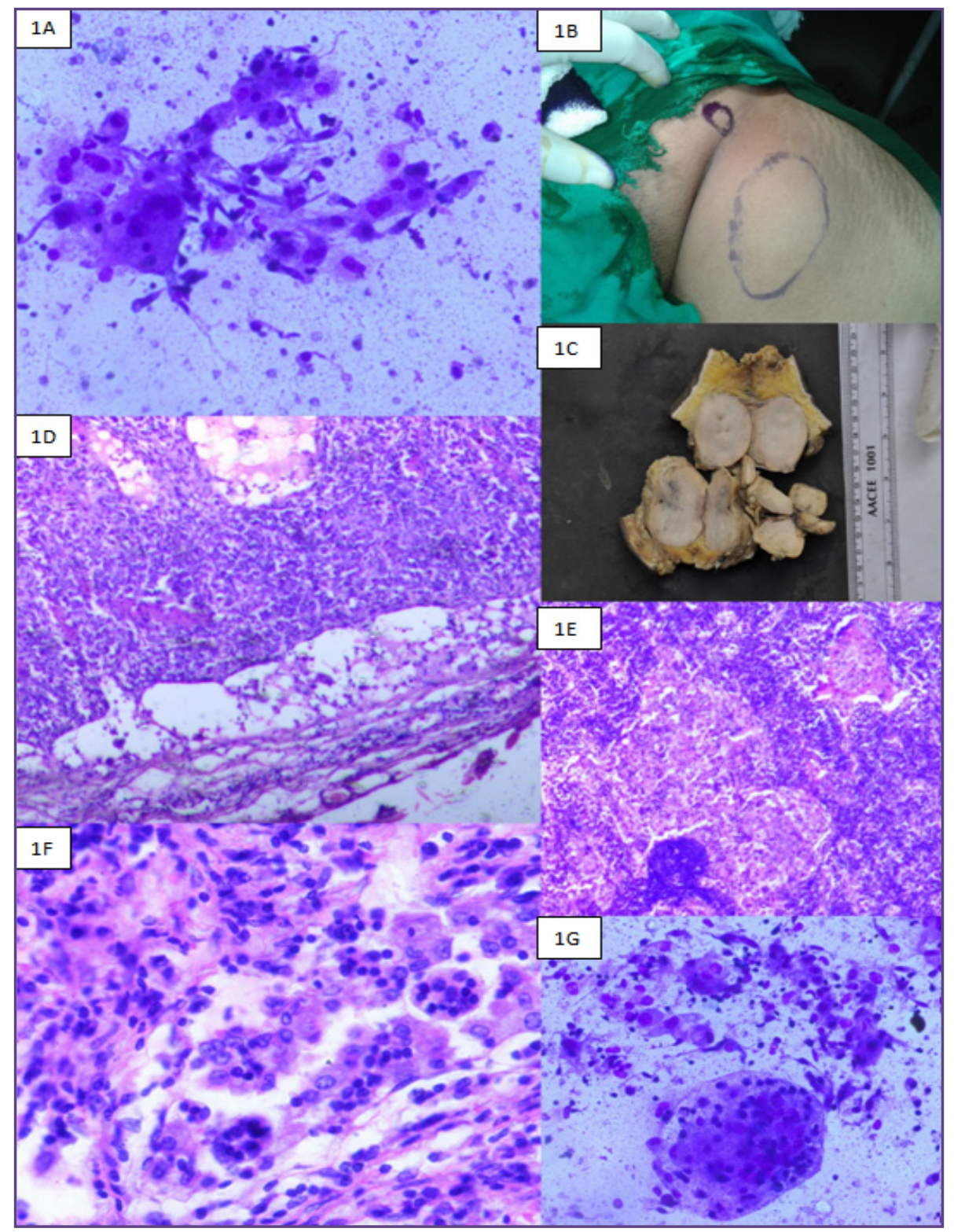

Fig. 1.A.Giemsa stained cytosmear from left thigh mass showing clusters of histiocytes and spindle cells;B. Swelling medial aspect of left thigh with normal overlying skin; C. Gross photograph of RDD showing well circumscribed multinodular subcutaneous mass with grey white fleshy appeareance. D. Thick fibrous capsule with dialated subcapsular sinus. (H \& E stain 10x); E. Dialated Sinuses with histiocytosis(H\&E stain, 100x); F. Emperipolesis (H\&E stain, 200x); G. Cytosmears showing emperipolesis on review (Giemsa stain, 200x). 


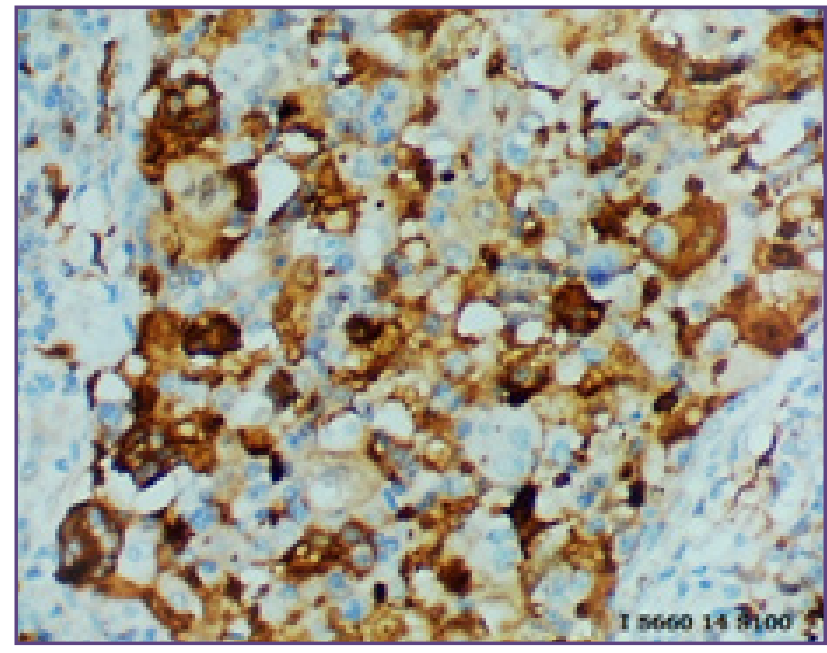

Fig. 2: IHC with S-100 showing strong positivity.(400x).

\section{Discussion}

Sinus histiocytosis with massive lymphadenopathy is a rare self-limited pseudo-lymphomatous disorder of unknown etiology. It is usually characterized by extensive, cervical lymphadenopathy, accompanied by fever, polyclonal gammopathy and leukocytosis with neutrophilia. ${ }^{[4,7]}$ The histopathological features of SHML were first described in 1965 by Destombes. Four years later, SHML was recognized as a clinicopathological entity by Rosai and Dorfman. ${ }^{[1]}$ By 1988, as it became evident that other nodal and extranodal sites can also be affected and the term Rosai-Dorfman disease (RDD) was preferred. ${ }^{[8]} \mathrm{RDD}$ affects all age groups, but predominantly people in the first and second decades of life. Males are slightly more susceptible to the disorder and it rarely affects people of Asian origin. ${ }^{[9]}$

RDD can be nodal or extranodal. Nodal RDD is seen in $87 \%$ of the patients and is often cervical, bilateral, massive and painless. Nodal RDD can also affect axillary (23.7\%), inguinal $(25.7 \%)$ and mediastinal (14.5\%) lymph nodes. ${ }^{[7]} \mathrm{RDD}$ involves both nodal and extranodal sites in $28 \%$ of patients. ${ }^{[8]}$ Histopathology and immunohistochemistry help to differentiate RDD from malignant disorders such as sarcomas, lymphoma and Langerhans cell histiocytosis. The classic histopathological features of RDD are dense diffuse or nodular infiltrates of foamy histiocytes, admixed with lymphocytes and plasma cells exhibiting emperipolesis. In emperipolesis, lymphocytes, plasma cells or neutrophils are captured but not digested by histiocytes. These specific types of histiocytes are called RosaiDorfman cells. The lymph nodes show capsular fibrosis and dilated nodal sinuses filled with foamy histiocytes that can exhibit emperipolesis. ${ }^{[9]}$ On immunohistochemistry it shows strong and consistent positivity for S-100 protein and for $\mathrm{CD} 68$, but not for $\mathrm{CD} 1 \mathrm{a} .{ }^{[10]}$ In the present case which presented as medial subcutaneous thigh swelling with clinical and cytological impression of malignant mesenchymal tumor i.e sarcoma. On histopathological examination, microscopic features suggested a diagnosis of Rosai Dorfman Disease which was confirmed by immunohistochemistry by S-100. It was strongly positive. On reviewing the cytosmears after histological diagnosis hallmark feature of Rosai Dorfman diseae emperipoliosis was demonstrated which was overlooked on cytological examination.

During literature review we found that all case reported as RDD had cervical, multiple or generalised lymphadenopathy or with cutaneous or extranodal manifestation. According to the literature reviewed no case with ingunal lymphadenopathy only was reported which was seen in present case.

\section{Conclusion}

RDD is a rare inflammatory non neoplastic process that should be considered in the differential diagnosis of lympadenopathy and soft tissue tumor such as soft tissue sarcoma. The diagnosis of RDD is made on the basis of clinical suspicion and is confirmed by histopathology and immunohistochemistry.

\section{Refrences}

1. Rosai J, Dorfman RF. Sinus histiocytosis with massive lymphadenopathy. A newly recognized benign clinicopathological entity. Arch Pathol. 1969;87(1):63-70.

2. Kong Y, Kong J, Shi D, Lu H, Zhu X, Wang J, Chen Z: Cutaneous Rosai- Dorfman Disease: a clinical and histopathologic study of 25 cases in China. Am J Surg Pathol 2007, 21:341-350.

3. Pinto DCG, Vidigal TA, Castro B, Santos BH, DeSousa NJA: Rosai-Dorfman disease in the differential diagnosis of cervical lymphadenopathy. Bras J Otorrinolaringol 2008, $74: 632-635$

4. Komaragiri et al.: Extranodal Rosai-Dorfman disease: a rare soft tissue neoplasm masquerading as a sarcoma. World Journal of Surgical Oncology 2013 11:63.

5. Foucar E, Rosai J, Dorfman RF. Sinus histiocytosis with massive lymphadenopathy (Rosai-Dorfman disease): review of the entity. Sem Diagn Pathol 1990;7:19 -73.

6. Ioachim HL, Medeiros J, eds. Ioachim's Lymph Node Pathology. 4th ed. Philadelphia: Wolters Kluwer/Lippincott Williams \& Wilkins; 2009.

7. Chang LY, Kou T, Chan HL. Extranodal Rosai-Dorfman disease with cutaneous,ophthalmic and laryngeal involvement: report of a case treated with isotretinoin. Int J Dermatol. 2002;41:888-91. 
8. Pitamber HV, Grayson W. Five case of cutaneous RosaiDorfman disease. Clin Exp Dermatol. 2003;28:17-21.

9. Landim FM, Rios Hde O, Costa CO, Feitosa RG, Rocha Filho FD, Costa AA. Cutaneous Rosai-Dorfman disease. An Bras Dermatol. 2009;84:275-8.
10. Becker M, Gaiser T, Middel P, Rompel R. Clinicopathologic challenge. Destombes- Rosai-Dorfman disease (DRDD) (sinus histiocytosis with massive lymphadenopathy). Int J Dermatol. 2008;47:125-7.

\section{*Corresponding author:}

Dr. Priyanka Patangia, Flat no. 2001,Type 2 : Block A Residential area, AIIMS Campus, Saket Nagar, Bhopal(India)

Phone: +91 9461656729

Email: drpriyanka1406@gmail.com

Financial or other Competing Interests: None. 